\title{
Finding Out the Possibilities of Improving OPD Services
}

\author{
Heenatimulla SDK*, Deepthi WKC, Samarawickrama N, Wijesinghe AK and Maranthota MRU
}

Outpatient Department, National Hospital for Respiratory diseases, Welisara, Sri Lanka

\begin{abstract}
Introduction: National Hospital for Respiratory Diseases (NHRD) at Welisara is the Premier tertiary care hospital in Sri Lanka for respiratory diseases. No studies have been done in its OPD set up to collect data which could have been invaluable for future planning and implementation. This study was done to gather such vital information in view of improving services rendered by NHRD OPD. Few parameters like socio-demographic details, distance to the hospital, cost for the visit, having a referral letter given by a doctor, number of persons accompanied the patient and reasons to visit NHRD were studied.

Methodology: This was a descriptive cross sectional study of patients who attended OPD of NHRD. Interviewer administered questionnaire was given to randomly selected 420 patients who were above 18 years of age and who were on their first visit to the hospital.

Results: Majority of patients were less educated and were coming from a low socio-economic background. Both sexes presented equally to the OPD. $68 \%$ of patients were coming from within $20 \mathrm{~km}$ of distance from the hospital and for $62 \%$, the cost for the visit was found to be 200 rupees or less. Only for $7 \%$ of people, NHRD was the nearest hospital. $95 \%$ of patients has presented with respiratory related conditions while only $2 \%$ had a referral letter with them. Most of the patients (around $80 \%$ ) were accompanied by 2 or 3 persons. More than $70 \%$ visited NHRD due to its reputation as the leading hospital in the country for respiratory diseases.

Conclusion: Middle aged less educated people with a low socio-economic status were the people who mostly visited the NHRD OPD. Most had respiratory system related presenting complaints. This is of significance and should be considered in giving health education to these patients. Majority of the patients resided close by to the hospital and their cost for the visit roughly showed similarities to the distances they travel. Though most patients visit NHRD after using nearby health facilities, lack of a proper referral system and accompained too many people can worsen the overcrowding at OPD. Strengthening the usage of nearby healthcare facilities can reduce this as NHRD was found to be the nearest hospital for very few of this population. Most common reason for visiting NHRD OPD was the reputation NHRD has gained as the leading hospital of the country for Respiratory disease, which should be conserved.
\end{abstract}

Keywords: Overcrowding; Respiratory diseases; Referral letter; Distance to the hospital

\section{Introduction}

Sri Lanka is a country in which the people are enjoying free health and education facilities, where the government is expending a significant amount of money for the provision of free health services and National Hospital for Respiratory Diseases (NHRD) at Welisara is the premier tertiary care hospital in Sri Lanka for respiratory diseases. It comprises of an Outpatients' Department (OPD), two surgical and fifteen medical wards. Currently there are three consultant chest physicians, three consultant cardio-thoracic surgeons, one consultant pathologists, one consultant radiologists and around seventy medical officers. Further, there are hundred and sixty nurses, sixty paramedical staff and support staff in NHRD.

The OPD comprises of twelve medical officers, twenty nurses, ten paramedical staff and ten support staff, provides its services 24/7, 365 days a year including all the public holidays. However, after $4 \mathrm{pm}$ on weekdays and after 12 noon on the weekends and public holidays, patients are not treated at the OPD still admitted to the appropriate ward after attending to their immediate problems.

Though NHRD is the leading hospital for Respiratory diseases in the country, no research has been done yet in the OPD setup of which data could have been helpful to overcome shortcomings in planning and implementation for the future betterment. Considering this shortfall of data on the OPD patients, demographic data like distance to the NHRD as well as a number of persons accompanying the patient and their income seems invaluable in getting an idea about the population benefited by the hospital.

As in other hospitals, NHRD OPD treats respiratory as well as nonrespiratory conditions. Respiratory illnesses are treated to the fullest while non-respiratory related conditions are referred to other relevant hospitals, mostly to the nearest hospital CNTH Ragama. As NHRD is advertised as a tertiary care institution specialized in respiratory diseases, its OPD follows the same path allocating most of its resources and manpower towards treating respiratory related diseases. Obviously, it is easier for the OPD staff if a high proportion of patients visit the hospital for respiratory related illnesses. So it is worthwhile to know the percentage of patients presenting to the OPD with respiratory / non respiratory illnesses to reevaluate the allocation of its resources for maximum productiveness.

In Sri Lanka, there is no established referral or back referral system in practice [1]. Due to the fame NHRD has gained as the leading hospital

${ }^{*}$ Corresponding author: Heenatimulla SDK, Outpatient Department, National Hospital for Respiratory diseases, Welisara, Sri Lanka, Tel: 94772009296; E-mail: sampathkdh@gmail.com

Received January 09, 2018; Accepted January 23, 2018; Published January 30, 2018

Citation: Heenatimulla SDK, Deepthi WKC, Samarawickrama N, Wijesinghe AK, Maranthota MRU (2018) Finding Out the Possibilities of Improving OPD Services. J Pulm Respir Med 8: 443. doi: 10.4172/2161-105X.1000443

Copyright: () 2018 Heenatimulla SDK, et al. This is an open-access article distributed under the terms of the Creative Commons Attribution License, which permits unrestricted use, distribution, and reproduction in any medium, provided the original author and source are credited. 
in the country for the respiratory illnesses, its OPD gets patients from all over the country. Even at the top of the tertiary care level, seeing a referral letter is rare. It's beneficial if the decision can be taken by a Medical doctor in referring for further investigations and management to NHRD rather than patients taking the decision themselves on visiting the tertiary care level. Obviously it is important to know the proportion of patients having a referral letter with them given by a doctor, to get an idea of the functioning level of proper referrals to NHRD.

Furthermore, the distance patients travel for the visit, the cost they have spent and the facilities they have used in the nearby hospitals and the reasons for visiting the NHRD are all very important information which has never been evaluated. These sorts of studies help to improve services in the hospital and to help take action to improve patient satisfaction. It is found in recent researches that service satisfaction can significantly improve patient's quality of life [2].

\section{Methodology}

This was a descriptive cross sectional study of patients who attend the Outpatient Department of National Hospital for Respiratory diseases (NHRD), Welisara. An absolute draining population cannot be mentioned as patients present to NHRD OPD from almost all over the country. Study duration was 6 months starting from February 2015 to August 2015. Study population consisted of patients attended on their first visit to NHRD who aged more than 18 years. Data collection was done on patients presented to the OPD from 8 am to $4 \mathrm{pm}$ which is said to be normal OPD hours. The study sample of 420 patients was selected randomly among the present population to the OPD. Interviewer administered questionnaire in English and Sinhala was used in these patients. The selected OPD patients were interviewed in the OPD waiting area before they were called to see the doctor. The doctor who interviewed the patient filled the questionnaire while interviewing. The patients who were unable or refused to respond due to the illness or any other reason were excluded from the study.

Data was collected only on working days where the OPD was opened till $4 \mathrm{pm}$. The patient to be given the questionnaire was considered before seeing the patient to minimize observer bias. Usually more patients visit NHRD OPD at the beginning of a week, on Mondays and the number reduces toward the end of the week on Fridays. Saturdays also have high numbers as more patients work on weekdays comes to the hospital on that day. But this can vary and the number of patients visit the hospital on a day could not be predicted. For this reason, a number 1 to 10 was picked randomly for a day and that number of patients was again selected randomly among the presenting population of patients that day. For example, if the first chosen number was 5, 5 numbers below 300 (during the period research was conducted, total number of patients were below 300) were selected randomly. If they were $17,33,39,168$ and 289 , patients had those numbers were interviewed. If the given number was not issued (for example 289) for that day, or when the patient with that number refused to participate in the interview, that number was neglected.

Informed written consent from every patient was acquired before proper interviewing process. Ethical clearance for the study was obtained from scientific and ethical review committee, Faculty of Medicine, University of Kelaniya. Written approval of the Director, NHRD Welisara was taken before starting the data collection process. Data were entered and analyzed using SPSS statistical software (GNU pspp 0.10.1-g6e73cb).

\section{Results}

The results of this study are presented under the names of the specific objectives below.

\section{Socio-demographic data}

An analysis of the general features of the sample population showed male to female ratio of $1: 1$. Of these OPD attendees, $86 \%$ were above 30 years while only $27 \%$ were above 60 years. In the population, $48.8 \%$ were not doing an occupation while $70 \%$ had their monthly income remained at 20,000 rupees or less category. The majority of the population hadn't passed their GCE O/Ls.

\section{Distance to the hospital}

For the patients, distance to the NHRD from their living place was as follows (Figure 1).

According to the values, nearly $50 \%$ of patients visited NHRD were from within $10 \mathrm{~km}$ from the hospital, giving similar results to the studies done at OPDs of other hospitals in Sri Lanka [3]. Another almost 25\% were from between $11 \mathrm{~km}$ to $20 \mathrm{~km}$, making it almost $68 \%$ patients to the NHRD OPD from within $20 \mathrm{~km}$ from the Hospital. Nevertheless, NHRD was the nearest hospital for only $7 \%$ of the population. Patients travelling more than $50 \mathrm{~km}$ for a visit at NHRD OPD were 5\%. (Skewed distribution, mean=21.07 and $\mathrm{SD}=25.27$ ) (Figure 2).

\section{Number of persons accompanying the patient}

Almost $50 \%$ of patients were accompanied by another person, while more than $80 \%$ of the patients were accompanied by 1 or 2 persons.

\section{Having a referral letter given by a Doctor}

It was striking to find that only 10 patients out of the sample of 420 patients had a referral letter given from a Doctor (Medical officer or a consultant). As a percentage, it was $2.38 \%$. During the study, verbal referrals were not counted as referrals.

\section{Cost for the visit}

The cost for the visit depends both on the mode of transport the patient has chosen to travel to the NHRD as well as the number of persons accompanying the patient for the visit. The costs for the visits were as follows (Figure 3).

More than $60 \%$ of the patients have had a cost of less than 200 rupees for the visit while $17 \%$ of them in the category had spent 50 rupees or less. But the average cost for a visit lies at 276 rupees for a visit due to the high cost some patients had to handle travelling long distances and by probably using other modes of transport than public transport. Of the 34 patients who spent more than 600 rupees, $12(2.8 \%)$ of the patients had spent 1000 rupees or more for the visit. (Skewed distribution, mean $=274.63$ and $\mathrm{SD}=346.51$ )

Though the most used mode of transport has not been incurred in this study, it is probable to be public transport as found in other OPD setups in Sri Lanka [3] and other developing countries [4].

\section{Respiratory related presenting complains}

Presenting problems related to respiratory system were considered as sneezing, runny nose, nasal congestion, frontal headache (sinusitis like), sore throat, cough, breathlessness, chest tightness, wheezing, pleuritic chest pain and haemoptysis. While these complaints were given the priority, other features of respiratory tract infections are also being considered like fever, fatigue, hoarseness, myalgia and facial pain 


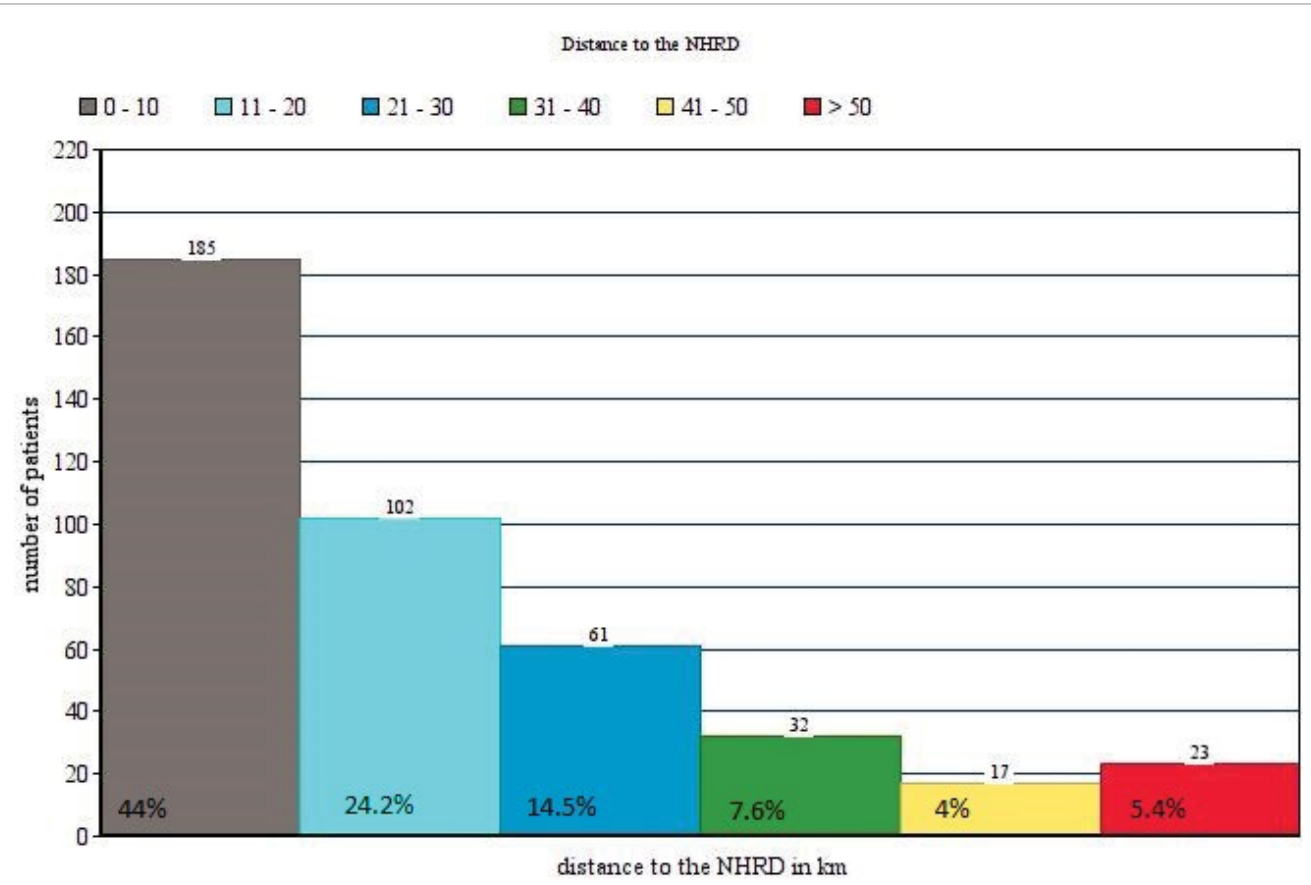

Figure 1: Distance to the hospital.

number of persons accompanying the patient

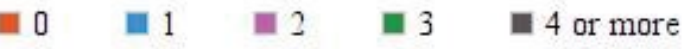

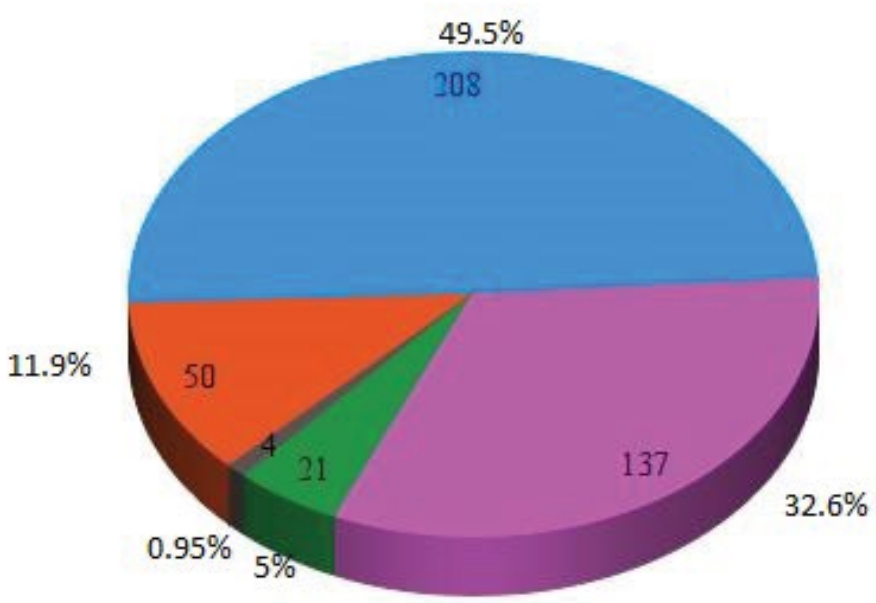

Figure 2: Number of persons accompanying the patient.

[5]. Some of the conditions needed some basic examination as well for confirmation of the condition [5]. Out of the 420 patients, only 19 patients $(4.5 \%)$ presented with non-respiratory related symptoms. All the other patients $(95.5 \%)$ had one or more of the above symptoms as their main presenting complaint (Figures 4-6).

\section{Reasons for attending NHRD}

Considering the visits to NHRD OPD, 131 patients (31.1\%) visited straight away without using any other nearby hospital facilities. Of them, $86 \%$ had visited NHRD due to its reputation as the leading hospital for respiratory diseases and it was the commonest reason for 

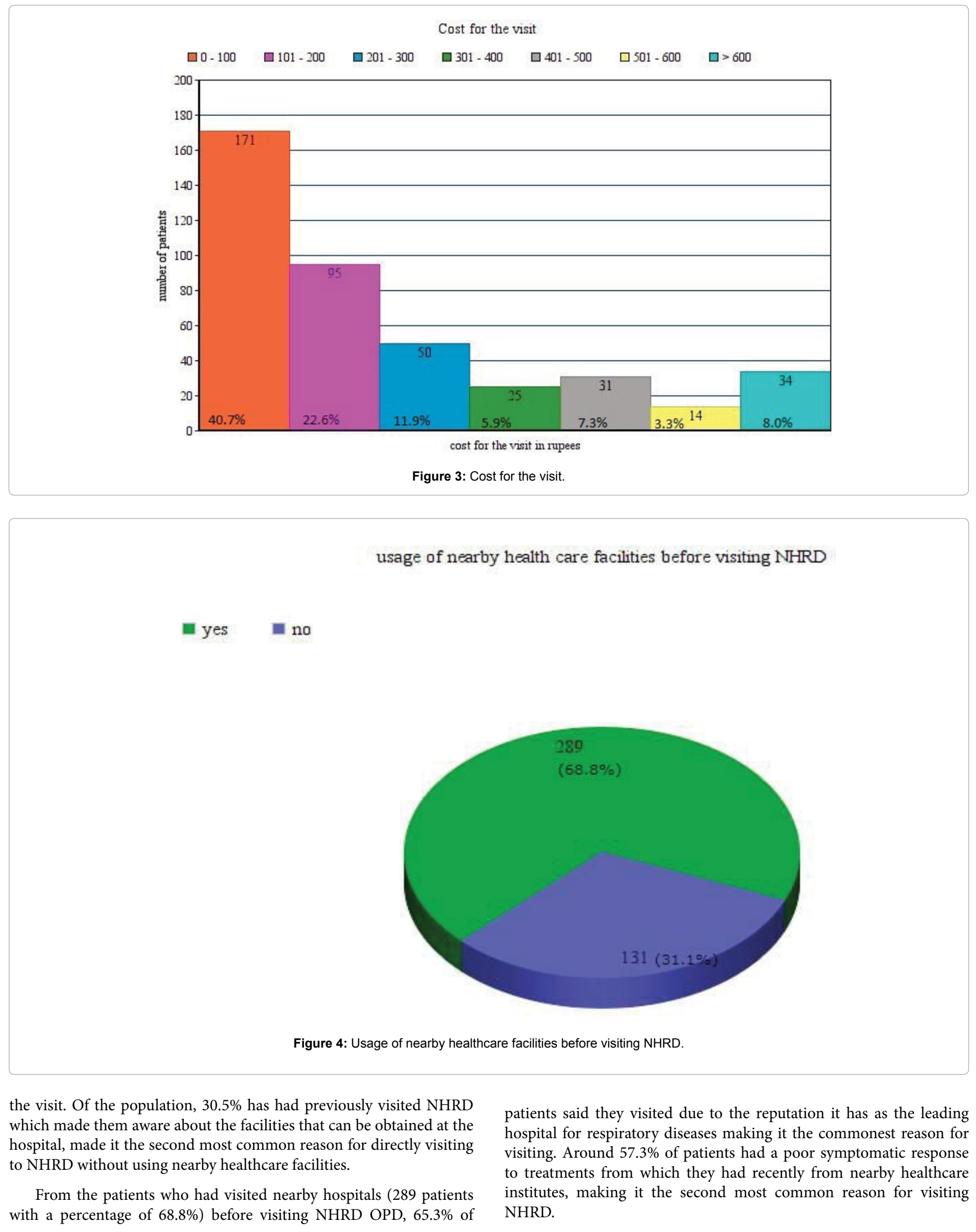


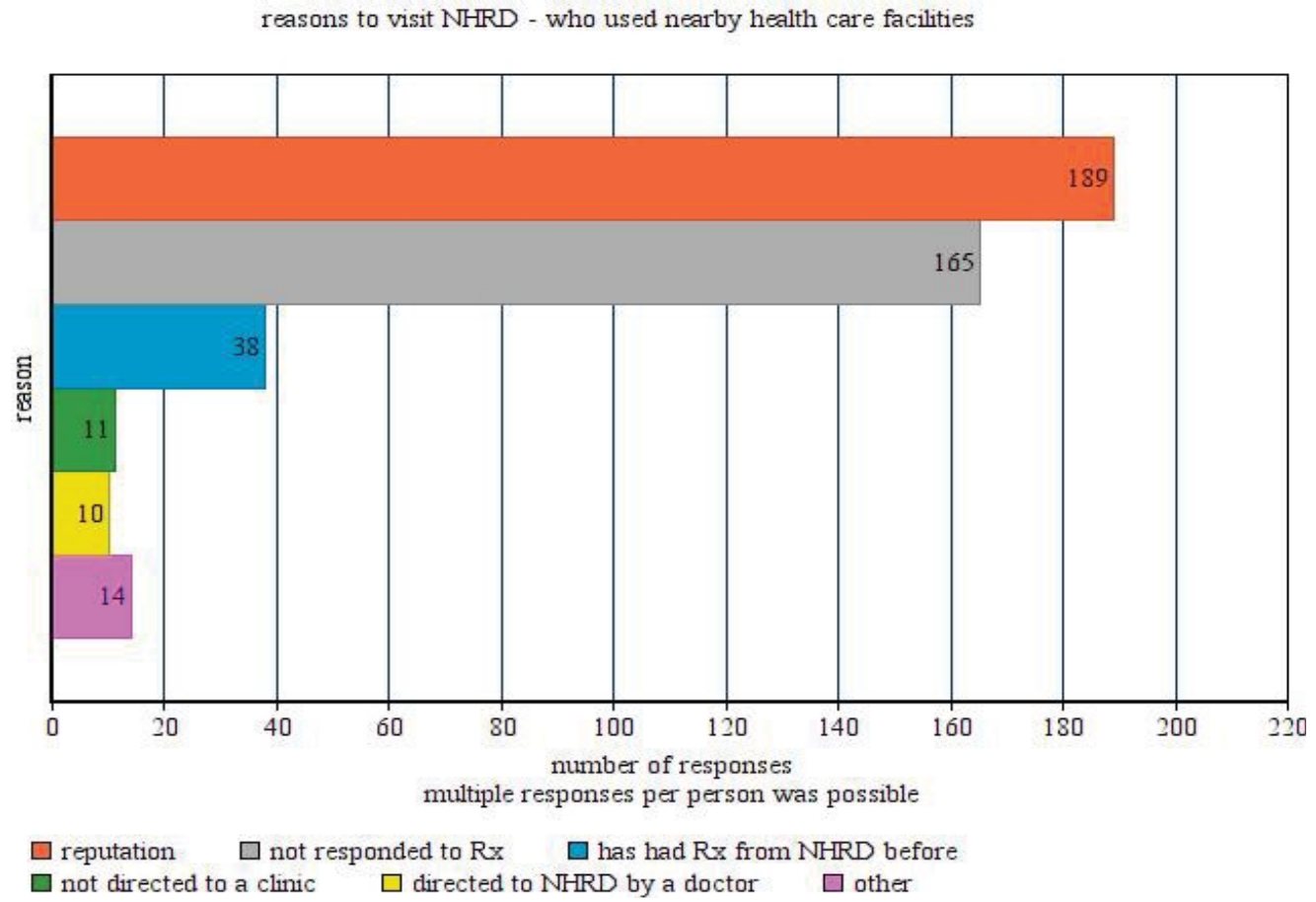

Figure 5: Reasons to visit NHRD-who used nearby healthcare facilities.

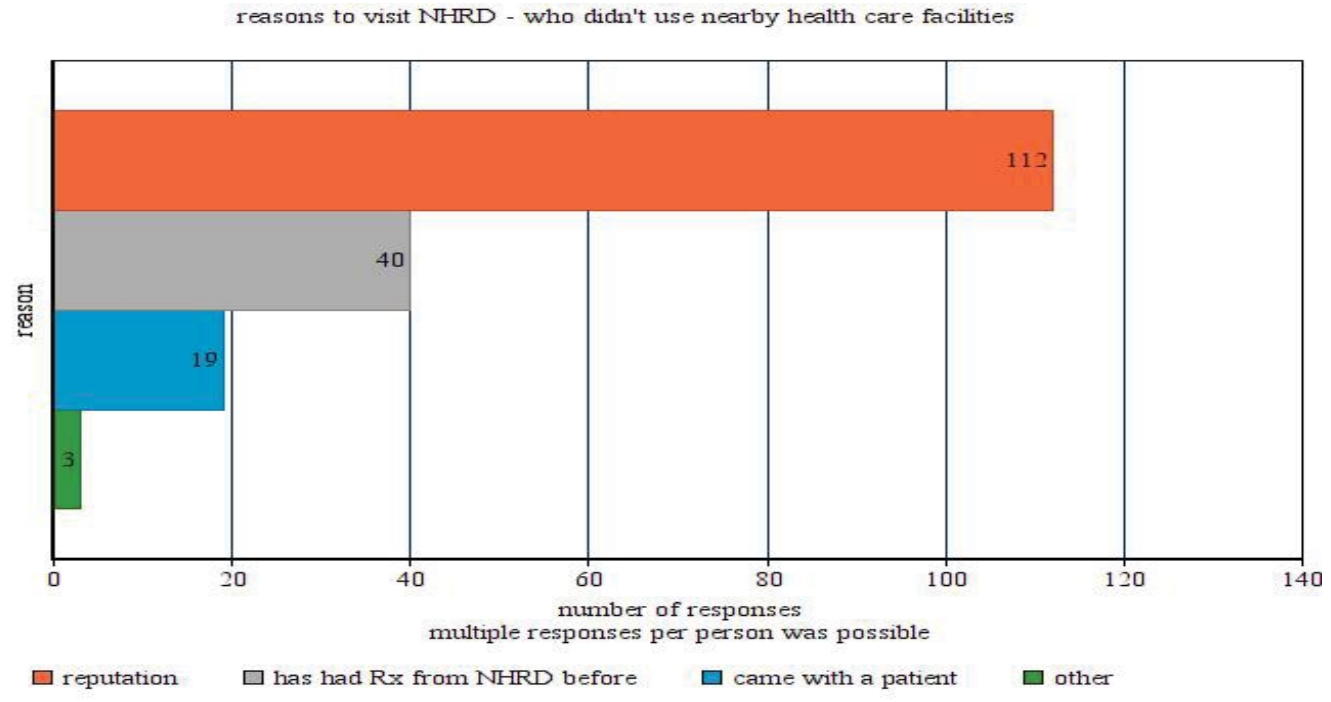

Figure 6: Reasons to visit NHRD-who didn't use nearby healthcare facilities.

\section{Discussion}

The study was conducted to find out the possibilities of improving the services rendered by the outpatient department of the NHRD Welisara. Considering the general features of the OPD attendees of NHRD welisara, both sexes had almost equal amounts presenting to the OPD. The majority were middle aged less educated people with a low monthly income, who are residing nearby to the hospital. This gave similar results to the studies done at OPDs of other hospitals in
Sri Lanka and in other developing countries, confirming that people who are less educated and with a low socioeconomic statuses are the majority who are using OPD services [3,4]. This makes a barrier to educate these patients regarding their conditions, arrange proper follow up as well as to maintain good compliance with the treatment. Moreover, it is imperative to give simple and easy to understand health material to these people as much as possible during health education. Giving expensive outside prescriptions should also be minimized as the cost can be unbearable for these patients. 
Looking into the findings on the number of persons accompanying the patient during a visit, it seems most of the patients are accompanied by 1 or 2 persons to the hospital giving similar results to studies done in different OPD settings of other developing countries [6]. Needless to say, this is largely responsible for the overcrowding that can be seen in the OPD setup. These persons accompanying the patient may or may not take treatment from the OPD and would be an interesting area for future researches. It is very important to categorize the patients from the accompanying persons who are not taking treatment, as it will help to release unnecessary overcrowding and queue at the OPD.

As the patients having a referral letter given by a doctor are less than $3 \%$, it depicts the frailty of the referring system to the hospital. Especially this is way below the expected when more than 50\% (39\% of all patients) of patients who already used nearby healthcare facilities visit NHRD due to lack of response to treatment. This scarcity of referrals can be due to many reasons like poor compliance and follow up due to the lack of knowledge about the condition, not waiting enough time to get responses from the treatment given, not informing the doctors at the first place regarding poorer response to treatment etc.

During the study, it was not considered a patient was referred when he/she was unable to produce any documents for the referral and verbal referrals were not counted as being done in other similar studies. This could have been a cause for the unexpectedly low levels of referrals in this study than studies done at the tertiary care level in other developing countries [7].

Lack of a proper referral system can also lead to overcrowding at the tertiary care hospitals $[7,8]$. For this reason, Usage of structured referral form among General practitioners to strengthen the referral system has been suggested by some of the studies done in Sri Lanka [1]. But there are many drawbacks to establishing a referral system for health sector in Sri Lanka as well [9].

Because most of the patients come to NHRD OPD are from nearby residences, the cost of the visit is below 200 rupees for the majority. But this amount of cost for the visit cannot be neglected as most of the patients visiting the NHRD OPD are holding a low socio-economic status. At the same time, $10 \%$ of the patients are spending more than 500 rupees for the visit shouldn't be disregarded as well because compared to their income, it can mean a lot for the majority of these patients.

As stated earlier, the best way to optimize the outcome and minimize the time and resource wastage, categorization of the patients at the stage of registration can be suggested. Measuring the waiting time of patients at the OPD would be beneficial to assess its outcome [6]. For the people coming to NHRD from long distances can be advised and educated regarding their nearby hospital facilities. Furthermore, it is also beneficial for these patients if they can be recognized earlier and treated appropriately. The significance of a quick referring back system to their nearby hospitals following early diagnosis and treatment should be contemplated.

It seems most of the patients visiting NHRD OPD are having respiratory related problems. Even without a properly functioning referral system, it lights some hope in the present situation. In this research, presenting complaints were incurred in relation to the respiratory symptoms and categorized as respiratory or non-respiratory related. As, this area of research data was subjective, there were instances where the classification was difficult. For instance, breathlessness was difficult to categorize as respiratory related or not as some patients gave a history that aroused suspicion on cardiac related breathlessness. At times, Sinusitis headache was difficult to distinguish from other common headaches like migraine and tension headache. In such situations, the data collecting person (Medical officer who collected the data at that time) used his/her medical knowledge to set it as respiratory related/non related. Though this division of the research seems to give some positive outcomes, it is important to assess the duration of these symptoms as well for a better completion of the analysis of this data, which sanguinely is open for future research.

The reputation of NHRD as the leading hospital in the country for respiratory diseases has been a major factor to guide the patients to the hospital, which seems to be a common finding for the tertiary care level [8]. There are roughly one third of patients appearing to the hospital without using any other close by medical facilities. If this amount can be reduced, it will be easier for the patient as well and will reduce the OPD overcrowding [8]. For this reason, patients can be educated regarding the availability of healthcare facilities and how to use them properly, during the morning health education sessions. Considering the target population, easy to understand healthcare material can further augment this process. Morning sessions on health education would be the most suitable time to give such information.

Contemplating on the data gathered majority of the patients paying a visit to NHRD OPD has had treatment from other hospitals/doctors which hasn't given an adequate response. It is imperative to identify these patients and close exploration into the history is necessary to get the relevant details about their recent treatments to avoid repeating the same treatment they had already.

\section{Conclusions}

- Majority of OPD attendees is residing nearby to the hospital Similarly, the cost for the visit was also low. Most of the patients were middle aged, having a low socioeconomic status and less educated. Both sexes visited the hospital OPD equally. Easy to understand materials should be used whenever possible in health education. Expensive outside prescriptions should be minimized as well.

- Most of the patients present to NHRD live nearby to the hospital. But NHRD is being the closest hospital only for a very few of them. This confirms that educating on improving usage of nearby healthcare facilities and re referring patients to their nearest primary and secondary care units can help in reducing burden on the hospital OPD.

- Most of the patients visiting NHRD were accompanied by 1 or 2 persons seems to be a main reason for overcrowding in the OPD. Categorization of patients from the people accompanying them will be very helpful in dealing with this issue.

- Even though the amount of patients visiting NHRD is quite a high following inadequate response to treatment that they had from nearby health services, only very few are having referral letters with them which delineating scarceness of referrals from local health services to NHRD. This area should be further explored and evaluated.

- Predominantly respiratory system related presenting complaints is presented to NHRD OPD. Non respiratory related complaints are very few in proportion.

- For the most part, reputation of the NHRD as the leading hospital for respiratory diseases in the country was the major cause that brought patients to the OPD. Most patients presented to NHRD OPD after having treatment from local healthcare facilities had poor response to treatment which made them visit NHRD. For 
Citation: Heenatimulla SDK, Deepthi WKC, Samarawickrama N, Wijesinghe AK, Maranthota MRU (2018) Finding Out the Possibilities of Improving OPD Services. J Pulm Respir Med 8: 443. doi: 10.4172/2161-105X.1000443

Page 7 of 7

this reason, previous history and treatments given should be explored thoroughly.

\section{References}

1. Ramanayake RPJC, de Silva AHW, Perera DP, Sumanasekera RDN, Lakmini KMS, et al. (2014) Referral communications: Bridging the gap between primary care doctors and specialists. Mid East J Family Med 12: 10-16.

2. Dagger T, Sweeney JC (2006) The effects of service evaluation on behavioral intentions and quality of life. J Service Res 19: 3-19.

3. Mediwaka MWMK, Karunarathna ADU, Wikramanayaka HMK, Jayasekara SAAN, Wijesinghe KDP (2014) A study on utilization pattern of Out Patient Department (OPD) at District General Hospital (DGH) Hambantota. Sri Lanka J Health Pol Manag 1: 5-11.

4. Pawan KS, Shaik IA, Manisha B (2008) Health care services in Punjab: Findings of a patient satisfaction survey. Social Change 38: 458-477.
5. Thomas F, Susanne F, Wolfgang H, Michael MK, Hummers-Pradier E (2008) Family practitioners' diagnostic decision-making processes regarding patients with respiratory tract infections: An observational study. Med dec making 28 : 810-818.

6. Tamiru Y, Wubegzier M, Emily JC (2013) Pre-post intervention study on overcrowding and waiting time at outpatient department in Amanuel Mental Specialized Hospital, Addis Ababa, Ethiopia.

7. Khalid UK, Somdatta P, Rohit S, Rupak S, Digamber B (2014) A study on overcrowding of out-patient Department of a Tertiary Care Tuberculosis Institute in Delhi. Indian J Prev Soc Med 45: 1-2.

8. Akande TM (2004) Referral system in Nigeria: Study of a tertiary health facility Annals African Med 3: 130-133.

9. Delpachithra C, Saroj J (2001) Overcrowding and "floor" patients in state hospitals: Institutions can make a difference. Ceylon Med J 46: 58-60. 